\title{
Image compression in digital video broadcasting
}

\author{
Kamil Bodecek, Vit Novotny, Milan Brezina \\ Faculty of Electrical Engineering and Communication, \\ Brno University of Technology \\ Purkynova 118, 612 00 Brno, Czech Republic \\ kamil.bodecek@phd.feec.vutbr.cz, novotnyv@feec.vutbr.cz, imb@email.cz
}

\begin{abstract}
Images form integral part of interactive services broadcasted in digital television. Natural images compression is always trade-off between visual quality and file size. Annoying artifacts arise in highly compressed images. The scope of this article is to investigate the possibilities of image compression enhancement for interactive services. The aim is to decrease size of the image file in such way that the following post-processing compensates the drop of the visual quality. A low-complex post-processing technique is evaluated. Proposed solution is suitable for decreasing downloading time of the images in digital video broadcasting services.
\end{abstract}

Keywords: mhp, dvb, jpeg, post-processing.

\section{Introduction}

In recent days there is a fast growing market in digital television. Together with digitalization, the interactive services have become available. With the term Interactive Digital Television (IDTV) the experts refer to TV with interactive content and/or digital enhancements. IDTV combines traditional TV watching with new interactive digital applications that may be developed to run on TV. Digital applications are broadcasted within a TV video stream by broadcasters and retrieved by Set Top Boxes (STBs) that execute them. These applications may be complementary to TV programs (e.g., voting systems, electronic program guides), or even completely uncorrelated to the program being watched by the user, thus representing new services exploitable while the TV program goes on (e.g., games, news, $t$-learning, $t$-commerce, delivery of Web contents).

\section{Interactive Digital Television}

Digital television in Europe is covered by Digital Video Broadcasting (DVB) project which defines global standards for the delivery of digital television and data services [1]. DVB was extended with generic, common application programming interface to enable interactive applications to be downloaded from DVB broadcast networks and run on STB receivers. The applications and the running environment of the STB are standardized by Multimedia Home Platform (MHP) which defines a generic interface

Please use the following format when citing this chapter:

Bodecek, K., Novotny, V., Brezina, M., 2007, in IFIP International Federation for Information Processing, Volume 245, Personal Wireless Communications, eds. Simak, B., Bestak, R., Kozowska, E., (Boston: Springer), pp. 477-487. 
between interactive digital applications and the terminals (STB, integrated digital TV) [2].

\subsection{MHP interactive applications and data}

An MHP application is an interactive application written in Java programming language. These applications are called "Xlets". The application model is very similar to the Applets from Web pages in that they are loaded and run by a life cycle manager, residing on the STB. DVB has adopted Java language and has created a lightweight version called DVB-J. Typical application design scenario is to separate application executive code and data content (text, images...), which is transported independently.

\subsection{Broadcast carousel - delivery of interactive data in DVB}

DVB networks are based on the MPEG-2 (Motion Picture Experts Group) standard [3]. MPEG-2 transport stream (TS) is form by multiple elementary streams containing video, audio, interactive data and service information, see Fig.1. Although MPEG-2 provides a means of transporting the Java application along with the audio-visual content, there is a problem in that the viewer may change channel and want to run Java program at any point in the transmission. Unless a user elect to view that program from the beginning, the application would already have been broadcasted. The result is that the STB and the viewer would have missed it.

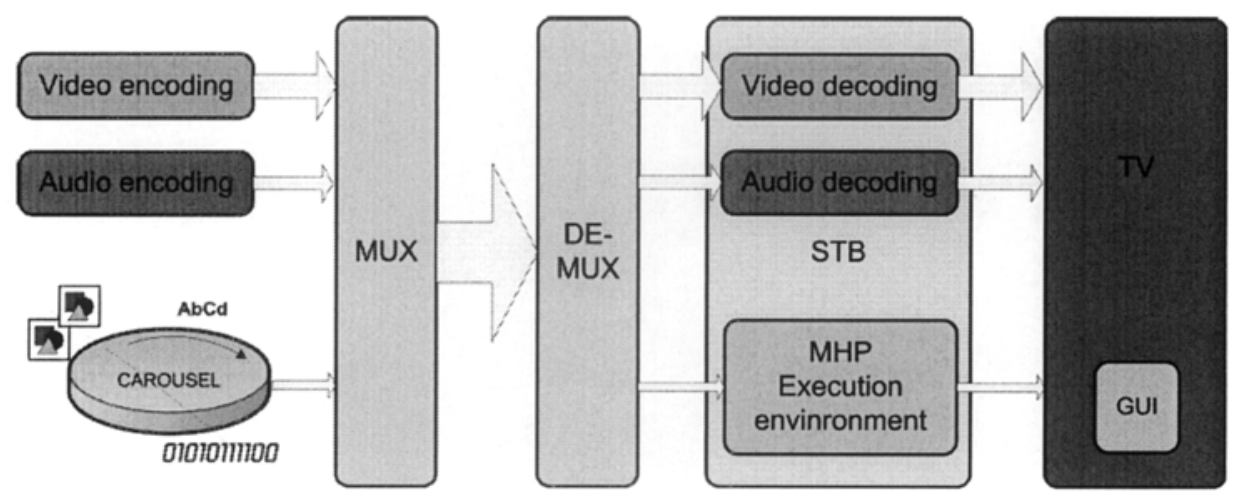

Fig. 1. Audiovisual and interactive data delivery in DVB.

The solution is in sending it over and over again so that the STB can pick up and assemble a complete application at any time during the transmission. This is provided by broadcast carousel (Digital Storage Media - Command and Control DSM-CC) - it keeps playing the same application around and around [4].

The packetized application is then continuously multiplexed with the audiovisual content for transmission. User can now join the transmission at any time and still have access to the interactive TV application. But it must be kept in mind that users can 
wait a full lap in the worst case to access a specific file unless the file is repeated during the lap. The longest access time for a file is defined by the bandwidth of the carousel and the size of the data in the carousel [5].

\section{Images in DVB-MHP applications}

Because of the visual character of the IDTV MHP applications, the images play important role in the application design and information transmission. The amount of the images in context with total size of the application and its data is very dependent on the type of the application. Images usually have large file size in comparison with the application code or text content.

The one of the goals of this paper is to investigate the influence of the perceived image quality of lossy compressed images in DVB-MHP applications.

\subsection{Image compression in DVB-MHP}

In MHP the image formats are JPEG and PNG as default for pictures. Because GIF format decoder implementation is left on the STB manufacturer so it is not recommended to use it for compressed pictures. When presenting Web pages on the STB using Web browser client the decoding of the GIF images should be possible. Besides these well-known image formats there is a possibility to use MPEG I-frames for compressed images.

\section{JPEG}

The most known image compression format in MHP applications is JPEG (Joint Photographic Expert Group). Officially, the JPEG corresponds to the ISO/IEC international standard 10928-1, a digital compression and coding of continuous-tone still images or to the ITU-T Recommendation T.81 [6].

JPEG is used for lossy compression of the natural images. It is a representative of the transform-based image codecs. Compression ratio is in range from 0.25 to 5 bit/pixel. Visual quality and compression efficiency is content dependent. Especially in low bit rates, the typical compression artifacts as blockiness and ringing became noticeable.

\section{PNG and GIF}

GIF (Graphics Interchange Format) is an 8-bit-per-pixel bitmap image format that was introduced by CompuServe company. It is lossless with exception that only 256 colors can be used. This limitation makes the GIF format unsuitable for reproducing color photographs and other images with continuous color, but it is well-suited for more simple images such as graphics or logos with solid areas of color [7].

PNG (Portable Network Graphics) is a bitmapped image format that also employs lossless data compression as GIF does. PNG was created to improve and replace the GIF format, as an image-file format not requiring a patent license. PNG supports 
palette-based (palettes of 24-bit RGB colors) or greyscale or RGB images [8]. Because these formats are lossless they are out of scope of this article.

\section{MPEG I-frames and video drips}

Besides familiar image formats the MHP receiver can use MPEG I-frames to display a full-screen image (such as a background image) or video "drips". In both cases, there is a need to be aware of a few limitations. Some receivers may use the hardware MPEG decoder for decoding I-frames and video drips. Decoding those image formats may disrupt any broadcasted video that is playing. This may be a short glitch while a frame is decoded, or it may last for the entire time the image is displayed, depending on the receiver.

Still images in the background layer will normally be MPEG I-frames. It is a MPEG-2 video sequence with only one frame. Video drip is a new content format that is pretty much unique to the digital TV world. The main aim of this format is to provide a memory-efficient way for displaying several similar images. Basically, it is a very short piece of MPEG-2 stream. The first thing in the file is an MPEG-2 I-frame that can be decoded and presented to the user. This is followed by one or more $\mathrm{P}$ frames, which are then decoded based on the preceding I-frame. This allows the decoder to update a static image in a very memory-efficient way [9].

\subsection{Degradation of decoded image}

Low bit rate image coding is essential for many visual communication applications. When bit rates become low, most compression algorithms yield visually annoying artifacts that highly degrade the perceptual quality of image and video data. When compressed at the same bit rate, images with more details usually degrade more than those with fewer details. The coding bit rate is another important factor that determines quality. In lossy compression, there is a trade-off between the bit rate and the resulting distortion.

The blocking effect is the most noticeable artifact associated with JPEG and MPEG compression standard. These blocking artifacts are square blocks with the edges aligned with the $8 \times 8$ regions processed via the discrete cosine transform block. Blockiness arises since each block is encoded without considering the correlation between adjacent blocks [10]. JPEG operates in spectral domain, trying to represent the image as a sum of smooth oscillating waves. Spectral domain is appropriate for capturing relatively smooth color gradients, but not particularly appropriate for capturing edges, so the "ringing" artifacts occur.

Generally speaking, the ringing effect occurs in all coding schemes that involve quantization in the frequency domain, see Fig.2. 


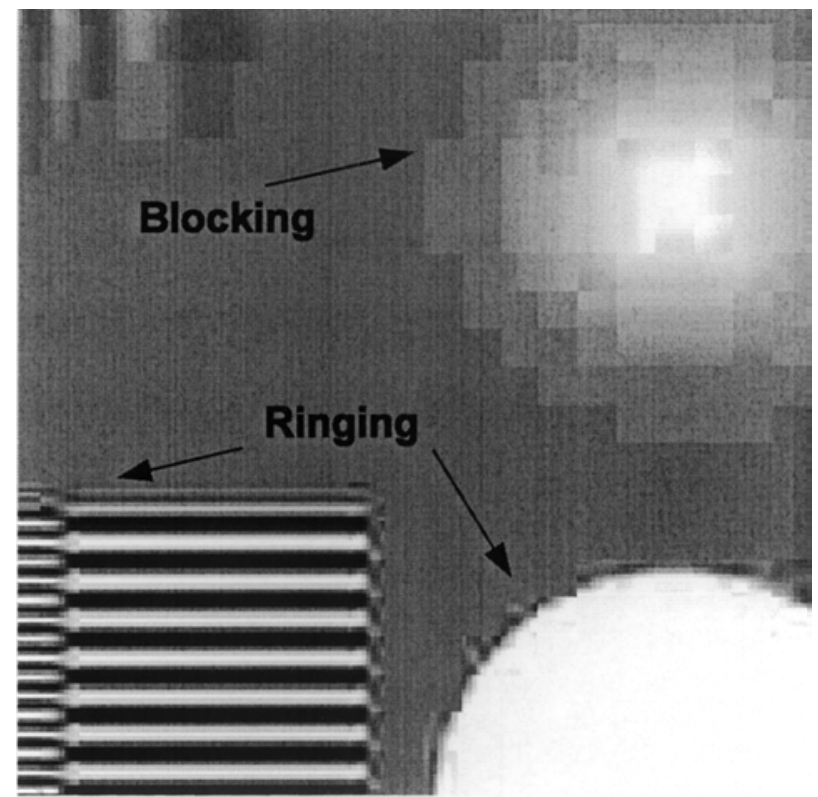

Fig. 2. Artifacts in JPEG image.

\section{Transmission efficiency improvements}

The second goal of this article is the evaluation of the proposals for image compression and/or transmission efficiency improvement. Firstly, it must be analyzed the multiplexed TS. For example, typical DVB-Terrestrial broadcasters' scenario is to have in one multiplex with $22 \mathrm{Mbits} / \mathrm{s} 4$ or 5 audiovisual services with additional data for Electronic Program Guide (EPG). The multiplex also contains MHP applications in broadcast carousel. Because the MHP applications are supplementary services complementary to broadcasted audiovisual service the carousel bit rate is quite small, in range from $100 \mathrm{kbits} / \mathrm{s}$ to $1 \mathrm{Mbits} / \mathrm{s}$. The size of the carousel data should not be too large. Long downloading time of the requested data is very annoying for the consumers. It could be expected that the waiting time should not be longer than 10 to $20 \mathrm{~s}$. Therefore the maximum size of the carousel is $2 \mathrm{Mbits}$ to $20 \mathrm{Mbits}$.

MPEG 1-frame and JPEG is the only standardized solution in MHP for natural images coding.

\subsection{Image post-processing}

The investigated idea is that the broadcasted JPEG images are transmitted with lower quality and the STB employs sophisticated post-processing method for image enhancement. The file size of the image is reduced so the amount of the broadcasted 
images is higher or the same number of images has smaller file size and downloading time of the images is shorter. The first requirement that must be accomplished is that the total time from user request for the image to the finished downloading, decoding and displaying is longer then total time of downloading time of more compressed image, decoding, post-processing and displaying, i.e.

$$
L_{1}+D+V \geq L_{2}+D+P+V
$$

where $D$ is decoding time, $V$ is displaying time, $P$ is post-processing time, $L_{1}$ and $L_{2}$ are downloading times. Therefore

$$
L_{1} \geq L_{2}+P
$$

because decoding and displaying time is the same for both cases.

\subsection{Overview of post-processing techniques}

The aim of post-processing is to reduce coding artifacts, like blockiness and ringing, whereby the objective and mainly subjective quality is improved. Post-processing methods can be divided into two directions:

- Algorithms based on image enhancement, it means subjective improving visual quality.

- Algorithms based on image restoration, it means objective recovering image as close to original as possible.

Another division can be done according to the compression used for images. Many post-processing methods have been developed for removing ringing and blocking artifacts in JPEG images. Existing methods for image enhancement JPEG compressed images are discussed. Recovering methods will be introduced very briefly.

\section{Searching boundaries in JPEG images}

Blockiness reduction using wavelet subband decomposition

This method uses wavelet-based decomposition which locates the blocky noise on the predetermined block boundaries of their corresponding subbands. The coefficients with high value in detail subbands are reduced by a linear minimum mean square error filter, which exploits the signal and noise characteristics [11].

\section{Blockiness reduction employing spatial filtering of the image}

Many approaches have been developed to reduce blockiness and ringing utilizing the local image characteristics and filtering methods driven by quantization parameters.

Blocking artifacts detection is based on variance differences within each $8 \times 8$ block. If the block boundary is recognized the 1D spatial low-pass filter is applied across the boundary. Then each pixel is classified according to a local variance value into three groups: smooth region, texture region and edge region. All pixels in block, where at least one pixel is classified as an edge pixel, are filtered by an adaptive fuzzy filter [12], [13]. 
A spatial filtering performed by an adaptive filters controlled by quantization parameters are also very often used. These filters can be linear or nonlinear. It must be noticed that nonlinear filter reduces the blockiness more effectively. A quantization parameter is important parameter for artifact reduction, because a new pixel value should not exceed the interval delimited by a quantization step.

Usually a 1D spatial filter is used for reducing the block artifacts and 2D spatial filter for reducing ringing artifacts [14].

\section{Iterative methods for image restoration}

Several classical image restoration techniques, including projection onto convex sets (POCS) and maximum a posteriori (MAP) restoration have been used for minimizing the compression artifacts. These methods are mostly very complex due their iterative execution.

Method using weighted combinations of shifted transforms is developed for deringing and deblocking [15]. A shifted transform is applied to the input image, then modified by a non-linear denoising point transformation and then inverse transform to the shifted transform is applied.

An N-point DCT is employed to obtain the local image characteristics and a $2 \mathrm{~N}$ point DCT is employed to obtain the global one. The comparison between N-point and $2 \mathrm{~N}$-point DCT coefficients makes the possibility to detect the high frequency components, which correspond with the block artifacts [16].

\section{Proposed algorithm}

All post-processing methods are computationally extensive. One of the lowcomplexity denoising methods has been introduced in [17]. This method simply reapplies JPEG algorithm to the spatially shifted versions of the already compressed images and forms an average. Although very simple, the effectiveness is at least comparative to nonlinear filtering methods, POCS and wavelet denoising methods. In that method, the forward and backward JPEG coding is performed 63 times and the result is average of these computations and received image. Only non-invertible portions of JPEG (DCT and quantization) are participated in the algorithm for speedup the processing.

In our experiments the simplified post-processing version of this algorithm has been evaluated. We have used only 3 shifted forward and backward JPEG coding as depicted on Fig.3. The drop of the improvement is trade-off between computational complexity and visual enhancement as can be seen on Table 1. 


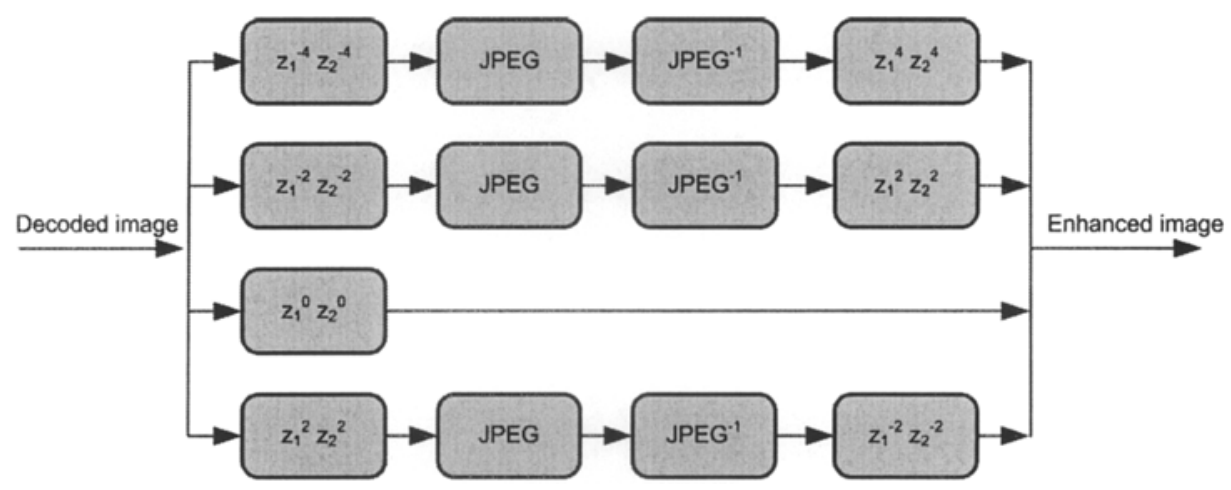

Fig. 3. Proposed algorithm of reduced re-application of JPEG.

Table 1. PSNR comparison for Lena image.

\begin{tabular}{|c|c|c|}
\hline \multirow{2}{*}{$\begin{array}{c}\text { JPEG PSNR } \\
{[\mathrm{dB}]}\end{array}$} & \multicolumn{2}{|c|}{ PSNR improvement [dB] } \\
\cline { 2 - 3 } & JPEG [16] & Proposed \\
\hline 26,65 & 1,17 & 0,83 \\
\hline 29,74 & 1,01 & 0,74 \\
\hline 32,34 & 0,65 & 0,64 \\
\hline
\end{tabular}

\section{Post-processing time requirements analysis}

Analysis of the computational load of the processor in the STB is based on assumptions defined in Eq.2. As a possible model of the carousel we have established broadcast carousel with bit rate of $200 \mathrm{~kb} / \mathrm{s}$. Lena image has been used in 3 different spatial resolutions and 1 cropped version of Bike image in greyscale. Quality factor 15 has been set in JPEG compression. After proposed post-processing, the new less compressed image has been created with objective quality equal to the original JPEG image. The size differences between original JPEG and Equal JPEG give the maximum available post-processing time which is dependent on the carousel bit rate as can be seen in Table 2. Visual comparison shows that there is some loss of sharpness in post-processed image in comparison with equal objective quality JPEG, but the subjective perceived quality has been improved, see Fig. 4. 
Table 2. Analysis of post-processing time

\begin{tabular}{|c|c|c|c|c|c|}
\hline Width & $\mathrm{px}$ & 720 & 256 & 512 & 512 \\
\hline Height & $\mathrm{px}$ & 576 & 256 & 256 & 512 \\
\hline Pixels & - & 414720 & 65536 & 131072 & 262144 \\
\hline PAL size & $\%$ & 100,0 & 15,8 & 31,6 & 63,2 \\
\hline Decoded & $\mathrm{dB}$ & 26,93 & 30,59 & 31,46 & 32,50 \\
\hline Bit rate & $b / p$ & 0,58 & 0,42 & 0,34 & 0,26 \\
\hline Size & $\mathrm{B}$ & 30315 & 3420 & 5549 & 9531 \\
\hline Processed & $\mathrm{dB}$ & 27,37 & 31,15 & 32,04 & 33,13 \\
\hline Equal JPEG & $\mathrm{dB}$ & 27,45 & 31,20 & 32,10 & 33,20 \\
\hline Size & $\mathrm{B}$ & 33948 & 3832 & 6222 & 10617 \\
\hline Size saving & $\mathrm{B}$ & 3633 & 412 & 673 & 1086 \\
\hline Carousel bit rate & $\mathrm{kb} / \mathrm{s}$ & \multicolumn{4}{|c|}{200} \\
\hline Delay & $s$ & \multicolumn{4}{|c|}{10} \\
\hline Processing time & $s$ & 0,142 & 0,016 & 0,026 & 0,042 \\
\hline
\end{tabular}

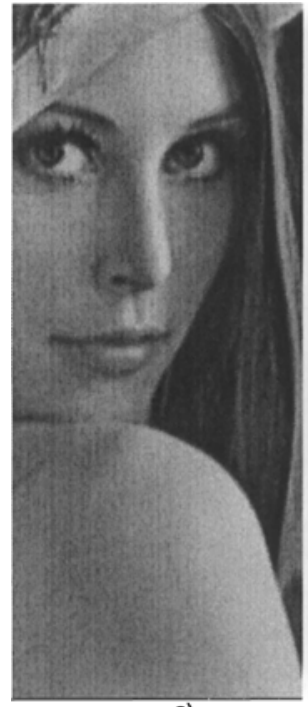

a)

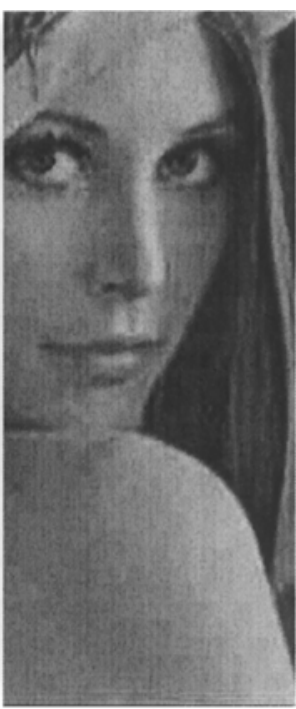

b)

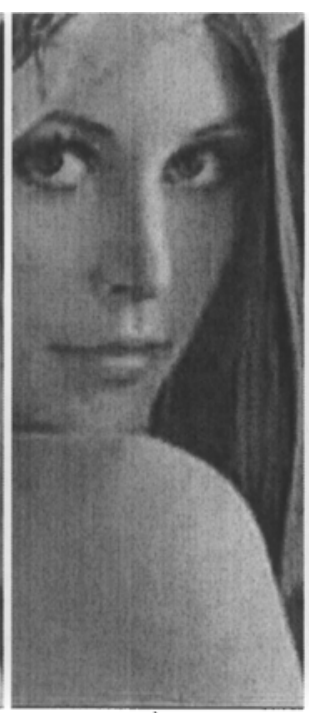

c)

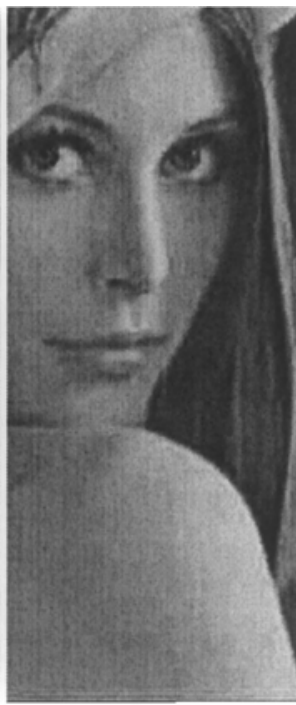

d)

Fig. 4. Post-processing of the Lena image: a) Original, b) Compressed (PSNR $=32,50 \mathrm{~dB}$ ), c) Post-processed $($ PSNR $=33,13 \mathrm{~dB}$ ), d) Equal objective quality JPEG without post-processing $(\mathrm{PSNR}=32,30 \mathrm{~dB})$. 


\section{Conclusions}

This paper presented image compressions standardized for DVB-MHP applications. In lossy compression there is a trade-off between visual quality and file size. Compression artifacts occur in highly compressed images. Short overview of postprocessing techniques is described and low-complex post-processing technique based on re-application of the JPEG algorithm proposed. The PSNR improvement is more then $0,6 \mathrm{~dB}$. Although there is some loss of sharpness in post-processed image in comparison with equal objective quality JPEG, the subjective perceived quality has been improved. Post-processing time speed-up and enhancement efficiency is still in subject of further research.

Acknowledgement. The research has been supported by Research Program No. MSM0021630513 and Academy of Science Project No. 1ET301710510.

\section{References}

1. DVB project website, URL: http://www.dvb.org.

2. Digital video broadcasting (DVB), Multimedia Home Platform (MHP) specification 1.0.3, ETSI Doc. No. ES 201812 V1.1.1, 2003.

3. ISO/IEC 13818: Generic coding of moving pictures and associated audio information.

4. ISO/IEC 13818-6:1998, Generic coding of moving pictures and associated audio information; Part 6: Extensions for digital storage media command and control, 1998.

5. Jones, J.: DVB-MHP/JavaTV Data Transport Mechanisms. In Proc. Fortieth International Conference on Technology of Object-Oriented Languages and Systems (TOOLS Pacific 2002), Sydney, Australia, pp. 115-121, Volume 10, 2002.

6. Wallace G. K.: The JPEG still picture compression standard, In Communications of the ACM, pp. 30-44, Volume 34, Issue 4, 1991.

7. Cover Sheet for the GIF89a Specification, URL:http://www.w3.org/Graphics/GIF/specgif89a.txt.

8. Portable Network Graphics (PNG) Specification (Second Edition) Information technology Computer graphics and image processing -Portable Network Graphics (PNG): Functional specification. ISO/IEC 15948:2003 (E), URL: http://www.w3.org/TR/PNG/.

9. Morris, S., Chaigneau, A. S.: Interactive TV Standards: A Guide to MHP, OCAP, and JavaTV. Focal Press, 2005.

10. Shen M.Y.; Kuo C.-.C.J.: Review of Postprocessing Techniques for Compression Artifact Removal, In Journal of Visual Communication and Image Representation, pp. 2-14, Volume 9, Issue 1, 1998.

11. Huyk Choi and Taejeong Kim: Blocking-Artifact Reduction in Block-Coded Images Using Wavelet-based Subband Decomposition, In IEEE Transactions on Circuit and Systems for Video Technology, pp. 801 - 805, Volume 10, 2000.

12. Hao-Song Kong, Yao Nie, Vetro, A., Huifang Sun and Barner, K.E.: Adaptive fuzzy postfiltering for highly compressed video, In Proceedings of International Conference on Image Processing ICIP '04, 24-27 Oct. 2004, Volume 3, pp. 1803 - 1806, 2004.

13. Hao-Song Kong, Vetro, A., Huifang Sun: Edge map guided adaptive post-filter for blocking and ringing artifacts removal, In Proceedings of International Symposium on Circuits and Systems, 2004. ISCAS '04, 23-26 May 2004, Volume 3, pp. 929-932, 2004. 
14. Hoon Paek, Rin-Chul Kim and Sang-Uk Lee: A DCT-Based Spatially Adaptive PostProcessing Technique to Reduce the Blocking Artifacts in Transform Coded Images, In IEEE Transactions on Circuit and Systems for Video Technology, pp. 36 - 41, Volume 10, 2000.

15. Samadani, R.; Sundararajan, $A_{s}$; Said, A.: Deringing and deblocking DCT compression artifacts with efficient shifted transforms, In Proceedings of International Conference on Image Processing ICIP '04, 24-27 Oct. 2004, Volume 3, pp. 1799 - 1802, 2004.

16. Hoon Paek and Sang-Uk Lee: On the POCS-based Postprocessing technique to Reduce the Blocking Artifacts in Transform Coded Images, In IEEE Transactions on Circuit and Systems for Video Technology, pp. 358 - 367, Volume 8, June 1998.

17. Nosratinia, A.: Denoising JPEG images by re-application of JPEG, In IEEE Second Workshop on Multimedia Signal Processing, 7-9 Dec 1998, pp. 611-615, 1998. 